\title{
Production of Bio-Polymer Structures by Soft Molding Method with Biomimetic Approach
}

\section{Melike ARSLANHAND, Murat EROĞLU®Cantekin KAYKILARLID Ebru Devrim ŞAM PARMAK*}

\author{
Departmant of Metallurgical and Materials Engineering, Bursa Technical University, 16310 Bursa, Turkey
}

Cite this paper as:

Arslanhan, M., Eroğlu, M., Kaykilarli, C., Parmak Şam, D.E. (2021). Production of Bio-Polymer Structures by Soft Molding Method with Biomimetic Approach. Journal of Innovative Science and Engineering. 5(1): $12-24$

*Corresponding author: Ebru Devrim Şam Parmak

E-mail: ebrudevrim.sam@btu.edu.tr Tel: +90 (224) 3003527

Received Date: 08/05/2020

Accepted Date: 28/09/2020

(C) Copyright 2021 by

Bursa Technical University. Available online at http://jise.btu.edu.tr/

\section{(c) (i) (8)}

The works published in Journal of Innovative Science and Engineering (JISE) are licensed under a Creative Commons Attribution-NonCommercial 4.0 International License.

\begin{abstract}
Biomimetic is the name of the approach that seeks sustainable solutions to the problems, taking the perfect functioning of nature for millions of years. The interest shown in biomimetic surfaces, inspired by multi-scale structures found in many plants and animals, is increasing day by day. Especially the unique wettability properties of the lotus leaf and rose petal. In this study, inspired by the structures of lotus leaf and rose petal, using the soft casting method with dental bio-polymer materials, structures with pillar dimensions of micron $(\mu \mathrm{m})$ and millimeter $(\mathrm{mm})$ were produced. These structures are replicated from two commercial products with different pillar lengths and different pillar shapes, mushroom and conical needle tips. Surface topographies of the replicated final products were analyzed by optical and stereo microscopes. Contact angles were tested to examine the wettability properties of the surfaces. According to the microscope results obtained, the demolding process, which is the riskiest step of the replication process, was successfully passed thanks to soft casting. Contact angle analysis showed that different pillar lengths and different pillar shapes changed the wettability properties of the replicated final product. The replicated mushroom-shaped micron-scale pillar structures exhibited a rose-petal effect and hydrophobic properties (approximately 1000) with only a single-scale configuration, while conical needle-shaped pillars of millimeter $(\mathrm{mm})$ scale did not show any specific wetabilitty property.
\end{abstract}

Keywords: Biomimetics, Rose-petal, Bio-polymer, Soft molding. 


\section{Introduction}

Human being is a part of nature. Since the day of its existence, humanity has found the solution to all kinds of problems in nature again. The order of nature works faultlessly thanks to the patterns and strategies that have been tested for millions of years. "Biomimetics" is the name of an innovative approach that looks for sustainable solutions to the problems encountered by taking this success of nature as an example.

Biomimetics consists of the two Greek words "bios" meaning "life" and "mimesis" meaning imitation. Additionally, the words "biomimesis", "biomimicry", "bionics", "biognosis" mean the same as biomimetics [1]. In summary, biomimetic is a creative technology adaptation that uses or mimics nature to improve human life [2]. The purpose of this technology is to create products, processes and new type of lifestyles that are well adapted to life on earth in the long term.

The order of nature is modeled to achieve the highest efficiency using the least amount of materials and least energy. Because, after billions of years of evolution, nature has learned how to use resources to acquire maximum performance, what is useful, what is proper and human being has produced many perpetual solutions for this [2, 3]. For all these reasons, it is an appropriate decision for scientists, engineers and designers to find nature, which is the oldest source of information in order to find solutions to problems. Today, materials produced thanks to biomimetics find a wide range of applications from nanoscience to robotics and transportation technology, from artificial intelligence to medicine and military applications. Biomimetic covers a wide range of research topics, affects various fields of application and is thought to have an outstanding scientific, social and economic influence on quality of life [4]. In addition, everyday designs inspired by nature pave the way for new and advanced technologies with features such as self-cleaning, recyclability, environmentally friendly, aesthetic, high strength and longevity, based on minimum material and minimum energy.

Biomimetics, which has become one of today's popular themes due to these advantages, is also very interesting in materials science. In particular, nature is inspiring in imparting hydrophilic or hydrophobic properties to the surface of the material. For example, the lotus flower, through the micro and nanostructures on its surface, its superhydrophobic (extremely waterrepellent) feature keeps it clean even in the muddiest waters (Lotus Effect). In addition, rose petals are superhydrophobic, while droplets of water firmly adhere to the surfaces even when the petals are turned or tilted upside down. Natural or artificial (replicated) micro/nanopatterned rough surfaces are known to be water repellent or hydrophobic [5]. On the contrary, smooth surfaces that attract water are hydrophilic. Both define the wettability properties of the surface.

The wettability properties of smooth and rough surfaces are the definition of a surface as hydrophobic or hydrophilic, depending on the contact angle between the liquid and the surface. The contact angle can be described as a quantitative measure of the amount of a solid which is wetted by the liquid. If the water drop shows a tendency to spread on the surface, that is, if the contact angle is less than $90^{\circ}$, this surface is called hydrophilic. If the water drop shows tendency to stay spherical instead of spreading, that is, if the contact angle is greater than $90^{\circ}$, this surface is also called hydrophobic. When the water drop spreads completely and the angle it makes with the surface is less than $5^{0}$, this surface is named as superhydrophilic. If the water drop stays almost spherically and the angle it makes with the surface is more than $150^{\circ}$, this 
surface is named as superhydrophobic. In general, there are three significant wetting theories to clarify the wettability of the surface. These are the Young, Wenzel and Cassie-Baxter models [6]. The Young equation supposes that the surface is completely smooth and the contact angle is designated by the interfacial energies between the solid-vapour, solid-liquid, and liquid-vapour phases (Figure 1).

The Young Equation: $y s v=y s l+\gamma l v \cos \Theta$

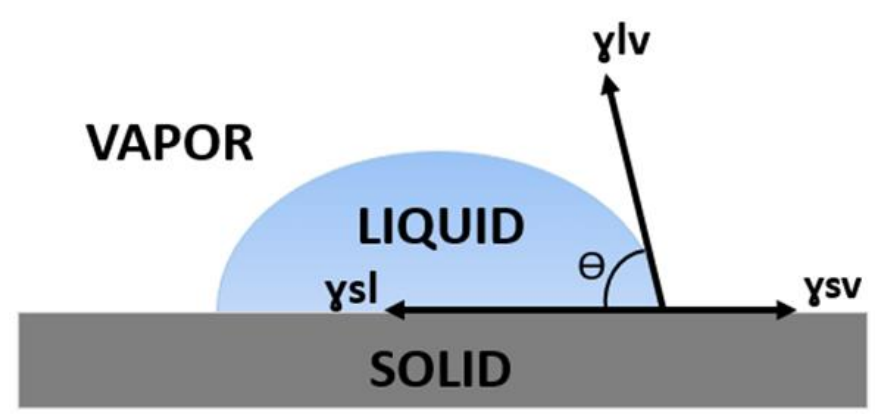

Figure 1. Contact angle ( $\Theta$ : contact angle, ysl: solid/liquid interfacial free energy, ysv: solid surface free energy, ylv: liquid surface free energy).

The Young model's disadvantage is that most solid surfaces are rough and this model is insufficient to explain the wettability behavior. Later, Wenzel developed the Young equation and proposed that the real contact area increased on a rough surface. In the Wenzel model, the liquid is acknowledged to be in full contact with the rough solid surface (Figure 2). Therefore, because of the larger contact area and stronger adhesion, it is hard for the water droplet to move and roll over the surface.

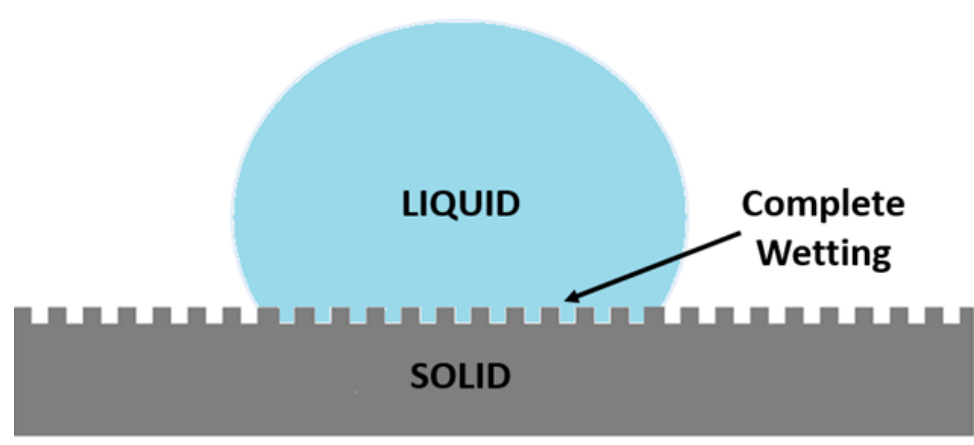

Figure 2. Wenzel Model

The Wenzel model defines homogeneous wetting with equation y.

The Wenzel Equation: $\cos \Theta \mathrm{w}=\mathrm{r} \cos \Theta \mathrm{y}$

where $\Theta w$ refers to Wenzel contact angle and $\Theta y$ refers to Young contact angle and $r$ is the roughness ratio [6, 7]. Besides, Cassie-Baxter Theory is the development of the Wenzel theory. Cassie- Baxter then offered a heterogeneous contact 
model in which the liquid on top of the rough surface, and the air pockets are trapped between liquid and solid [6]. The air pockets push the liquid upwards and the combination of solid-air-liquid interface is formed as shown in Figure 3.

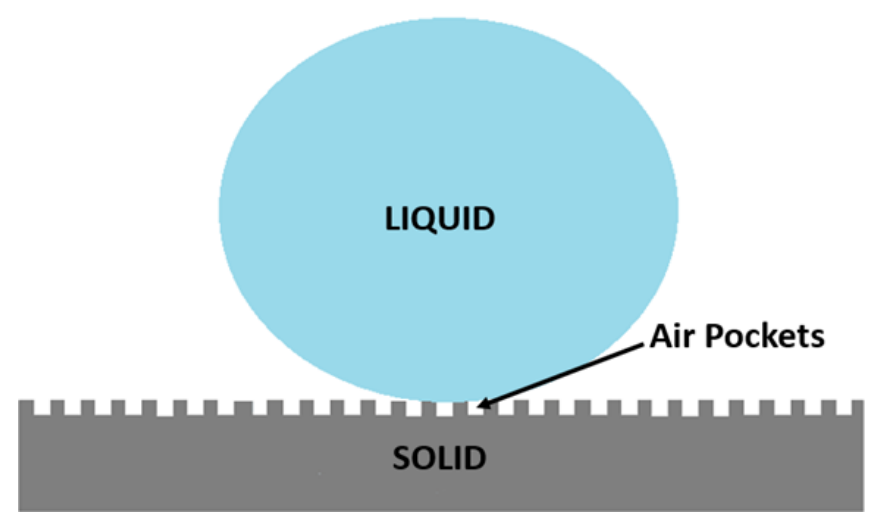

Figure 3. Cassie-Baxter Model

The Cassie model defines heterogeneous wetting by the equation $\mathrm{z}$.

The Cassie-Baxter Equation: $\operatorname{Cos} \Theta \mathrm{c}=r f \cos \Theta \mathrm{y}+f-1$

where $\Theta c$ refers the Cassie contact angle $\Theta y$ refers the Young contact angle, $r$ is the ratio of the real area to the estimated area of the solid surface that is wetted by the liquid, and $f$ is the area fraction of the estimated wet area [6, 7]. Respect to the details of the contact angle issue, Wenzel's condition can induce a high contact angle and Cassie's status can induce a low contact angle.

As stated in the theories, one way to increase the hydrophilic or hydrophobic properties of the surface is to change the roughness. For this reason, rough surfaces have been an important subject of hydrophobicity research. The most basic and simplest method to determine the wettability properties of a surface is dropping a liquid and observing with naked eye [6, 8]. The drop on the surface creates a contact angle based on the wetting property and the surface wettability can be examined by measuring the contact angle.

Several techniques for the fabrication of biomimetic multi-scale structures exist such as photolithography [9-15], the Capillary-Force Lithography (CFL) [15-17], Anodization [18-25] and soft-molding [26, 27]. In this study, a simple soft molding method was used for structuring the bio-polymer surfaces. The reason for choosing the soft molding method in this study is that the template is fully filled in the micro- and nanostructures and easily separated from the template. In addition, this technique is a low cost fabrication. Soft molding offers complex tip shape fabrication with high resolution consists of techniques based on self-assembly and replication molding of organic molecules and polymeric materials [26]. Thanks to the soft molding technique, polymer millimeter and microscale structures were successfully demolded from a polymer mold with similar hardness properties without being damaged because demolding is the riskiest stage of the casting methods. 
During the demolding process, the mold and sample material should be selected appropriately to avoid high adhesion forces that could lead to sample defects. Otherwise, a chemical interaction may occur between the sample and the mold. Therefore, the sample will not be separated from the mold as desired. In order to ensure that the cavities in the mold are completely filled, the liquid sample material should be selected correctly according to its viscosity, wetting property and air permeability properties. Otherwise, the mold is not completely filled and the sample with the desired geometry cannot be obtained. The sample material must be flexible enough to come out of the mold cavities without breaking, depending on the geometry of the mold. If the material is harder, the structures break in the demolding process and the desired structure does not occur [27]. The reason why dental bio-polymer material is preferred in this study is that the dental biopolymer material is in liquid form at the beginning of the application. Thanks to this liquid form, it fills the mold completely and converts to solid form in a short time. In addition, thanks to its flexible structure, it can be easily separated from the mold after drying. For all these reasons, the ideal mold material to be used in this study is polyurethane polymer, while the mimicking material is a dental bio-polymer.

\section{Material and Methods}

In this study, two commercial products were selected to use as template. These are a Velcro tape with mushroom-shaped pillars, each approximately $0.1 \mathrm{~mm}$ length named "PM" (Figure 4) and a commercial polymer facial cleansing pad with conical needle-shaped pillars with a length of about $0.4 \mathrm{~mm}$ named "PN" (Figure 5).

Firstly, the Velcro band and face cleaning pad were cut to a specific size. In order to prevent leakage of the liquid urethane polymer to be poured on, a barrier made of soft play dough was made around the cut pieces (Figure 6). Then, in order to replicate the voids ( $\mathrm{mm}$ and $\mu \mathrm{m}$ scale structures) present on the mold surface, a liquid polyurethane polymer (SmoothOn/Vytaflex 50) was spilled into the mold and left for 24 hours at room temperature for curing.

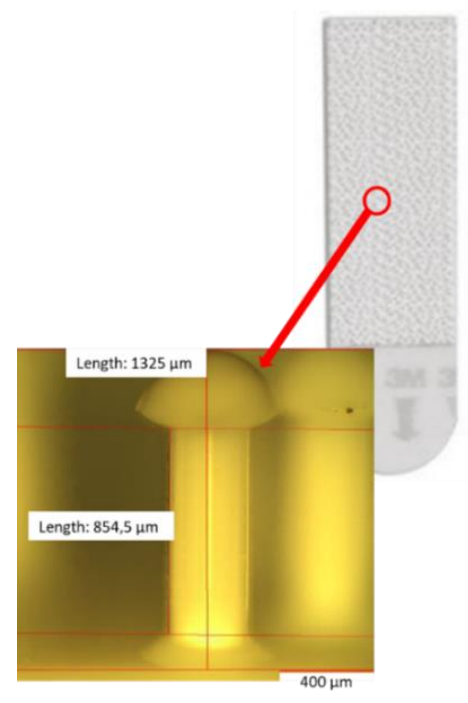

Figure 4: Velcro adhesive with mushroom-shaped pillars (back) and optical image of mushroom-shaped pillars (in front). 


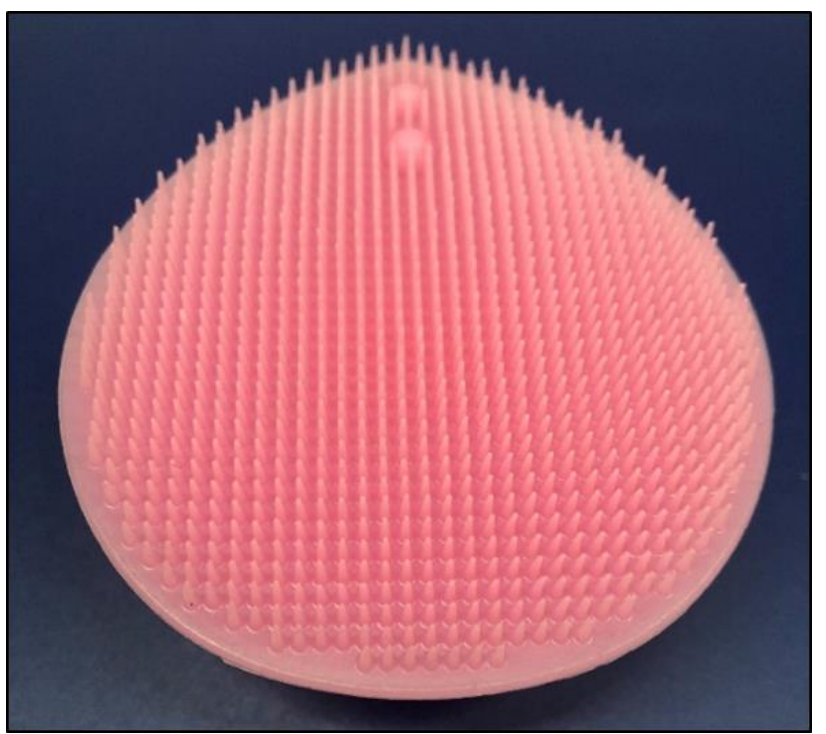

Figure 5: Polymer facial cleansing pad with conical needle-like shapes.

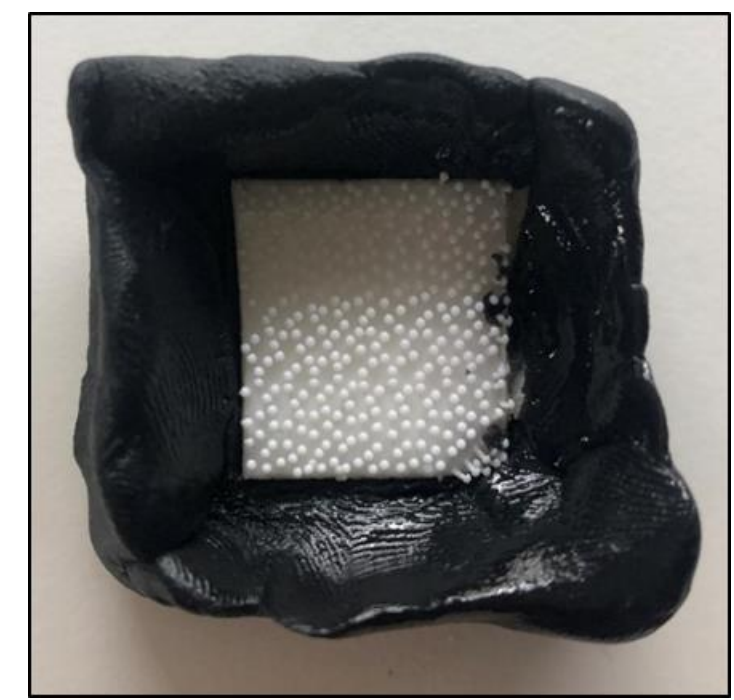

Figure 6: A barrier made of soft play dough.

The resin and hardener of the polyurethane were mixed in a 1: 1 ratio for at least 3 minutes. Afterwards, the templates were treated with a Teflon-like film to ensure good detachability of the template patterns from the polyurethane mold produced. The liquid urethane was then slowly poured onto the templates, minimizing air gaps between the pillars. They were left to be cured at room temperature for 24 hours. The soft play dough barrier around the dried polyurethane mold was carefully cleaned. Then, the Velcro adhesive and the polymer facial cleansing pad, whose pillar structure was replicated, were slowly separated from the polyurethane negative mold (Figure 7). 

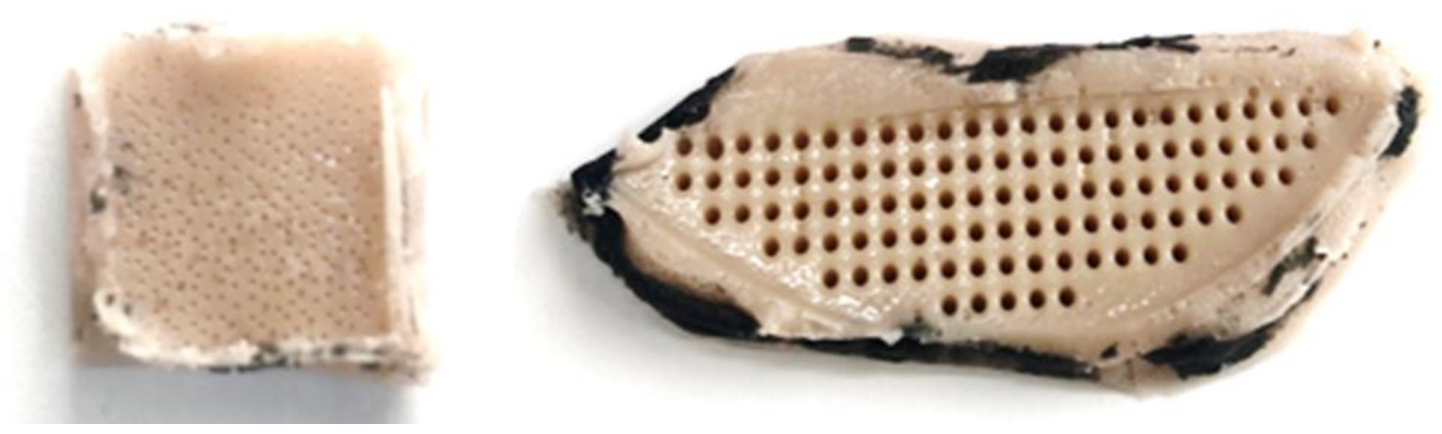

Figure 7: Polyurethane negative molds obtained in $\mathrm{mm}$ and $\mu \mathrm{m}$ scales

A schematic illustration of the production procedure was shown in Figure 8.

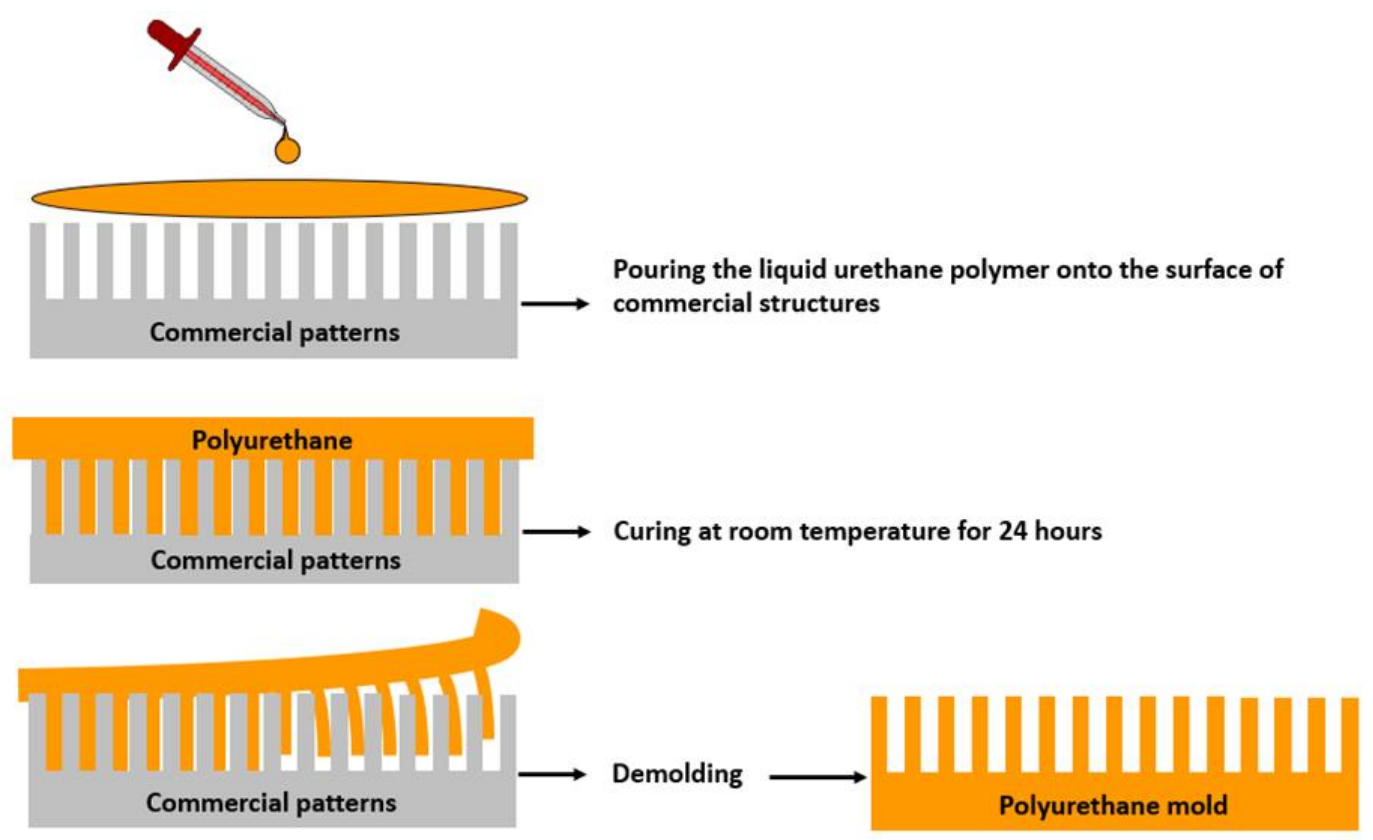

Figure 8: Schematic illustration of the polyurethane mold production

Resultant polyurethane molds were filled with a biocompatible dental silicone material (Zhermack/ Zetaplus). The reason for choosing this silicone material, besides being biocompatible, is the high flexibility it offers while preparing process and the appropriate hardness it reaches after drying. In addition, this silicone material can easily fill the pillar voids and after drying it can be easily demolded from the mold. The silicone material and catalyst were mixed in a proper amount as a viscous form and quickly poured into the negative mold. When the material was dried and hardened (room temperature/24 hours), structures were demolded from the polyurethane negative mold (Figure 9). 


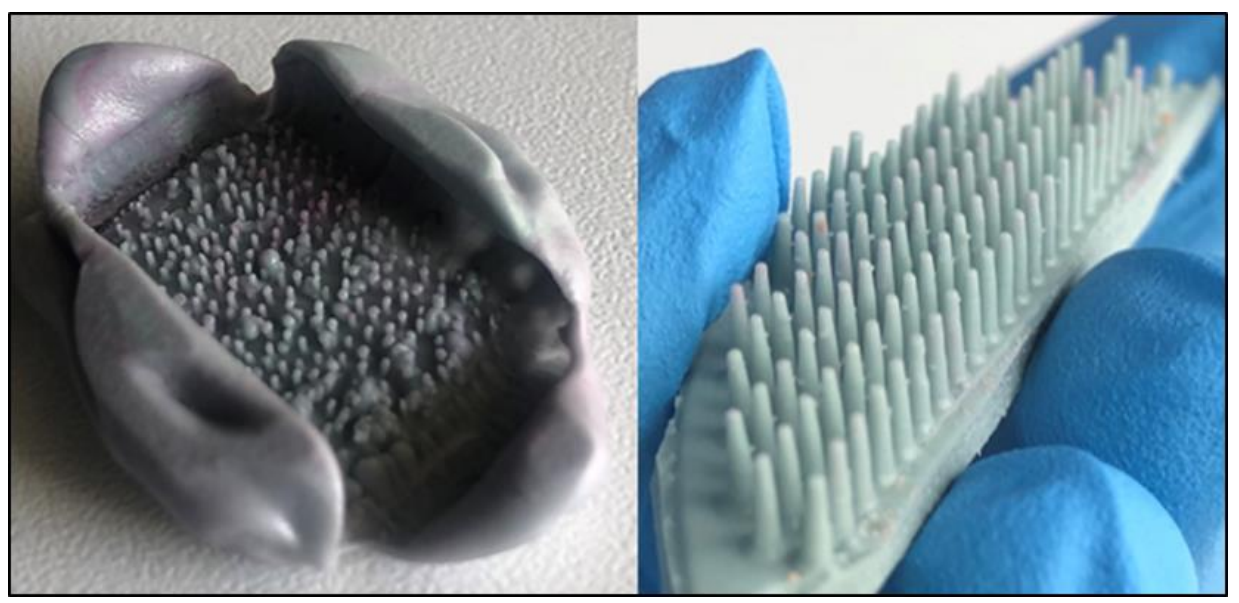

Figure 9: Replicated materials. Mushroom shaped (left) (PMR), conical needle shaped (right) (PNR).

After the replication process was completed Nikon-Eclipse LV $150 \mathrm{~N}$ model optical microscope was used for imaging patterns. So as to see the distribution of the structures on the sample surface a LIC-BM2500 (Stereo + Upright) model stereo microscope was used also. Contact angles of the resultant surfaces were measured by an Attension / Theta Lite model optical contact angle measuring device.

\section{Result and Discussions}

The polyurethane material has fulfilled all the requirements expected from the mold. One of the polyurethane molds was prepared using a commercial Velcro tape with mushroom-shaped pillars. The difficulty in replicating such mushroomshaped structures is the demoulding step. If the mold material is not flexible, the head of the mushroom-shaped pillars could be torn while demolding it from the mold. For this reason, both the selected flexible polyurethane material and the anti-sticking layer enabled the produced negative mold to be removed from the templates easily and without damage.

The dental bio-polymer material prepared in the viscose form is carefully spread over the polyurethane negative mold. Dental bio-polymer material, which started to solidify in a short time, was left to cure for 24 hours at room temperature. After curing, the dental bio-polymer material was demolded from the polyurethane negative mold slowly and carefully. Because, as noted previously, demolding is the most riskiest stage of the casting methods and the hardness of polyurethane mold (Shore A 50) and dental bio-polymer (Shore A 60) are close to each other. It is difficult in casting methods to easily separate materials with similar hardness from each other. According to the first observation made with the naked eye, this stage was successfully passed (Figure 9). Subsequent microscopic analyzes support this view. The optical microscope images presented in Figure 10 and Figure 11, the mushroom-shaped structures were demolded from the flexible polyurethane mold preserving their shape integrity. 


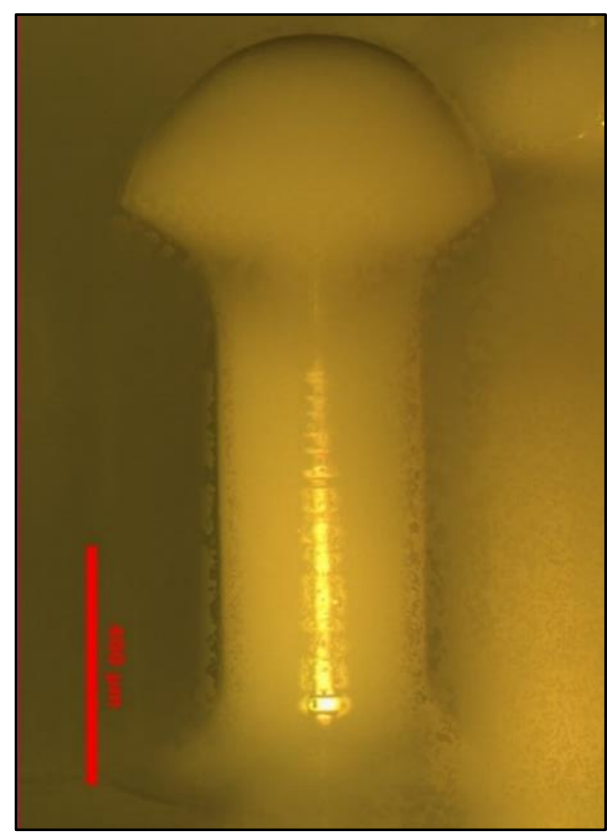

Figure 10: Optical microscope image of mushroom-shaped structures successfully removed from the polyurethane mold

Also, the conical needle -shaped structures were demolded from the flexible polyurethane mold preserving their shape integrity with high resolutions. Since the pillars of replicated conical needle -shaped structures are in the order of mm, their wettability properties were not examined.
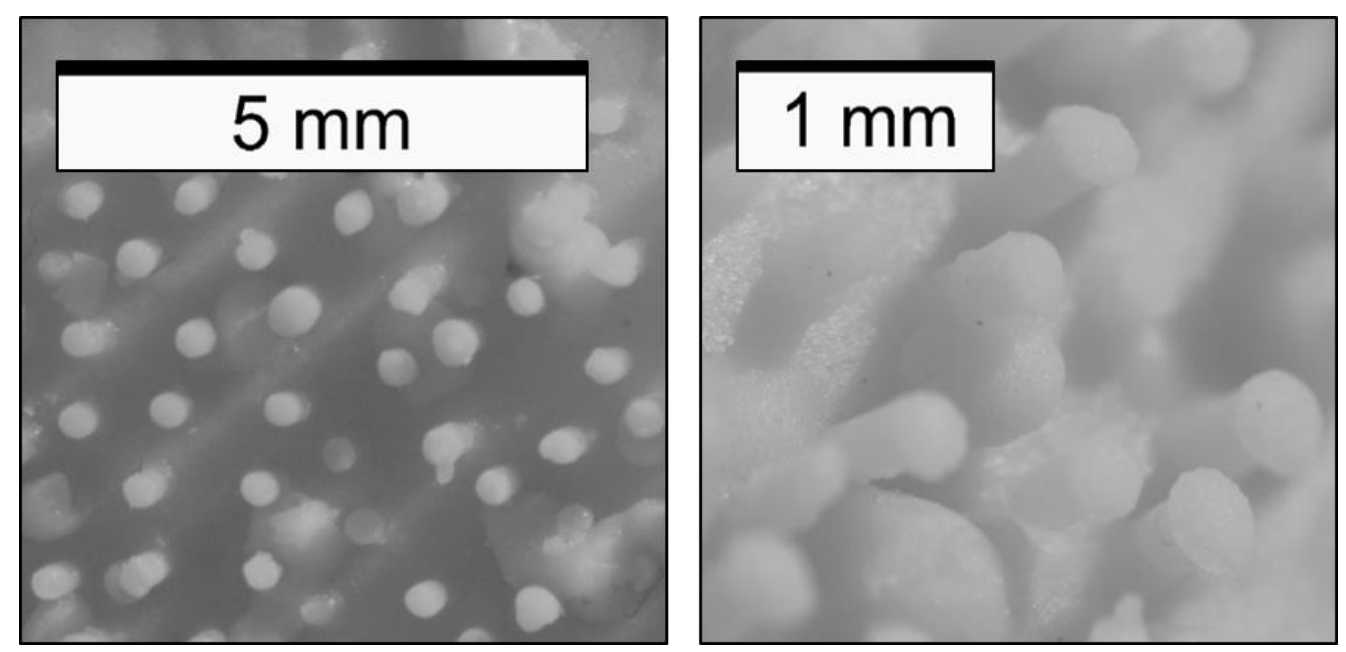

Figure 11: Stereo microscope image of mushroom-shaped pillar structures and dispersion on the surface of dental silicone material.

Contact angle measurements were made to determine the wettability properties of dental silicone structures. Previously, contact angle measurements of commercial products used as molds were made. The static contact angle of the mushroomshaped pillars was measured as $120^{\circ}$ (Figure 12) on average after about 3 measurements. 


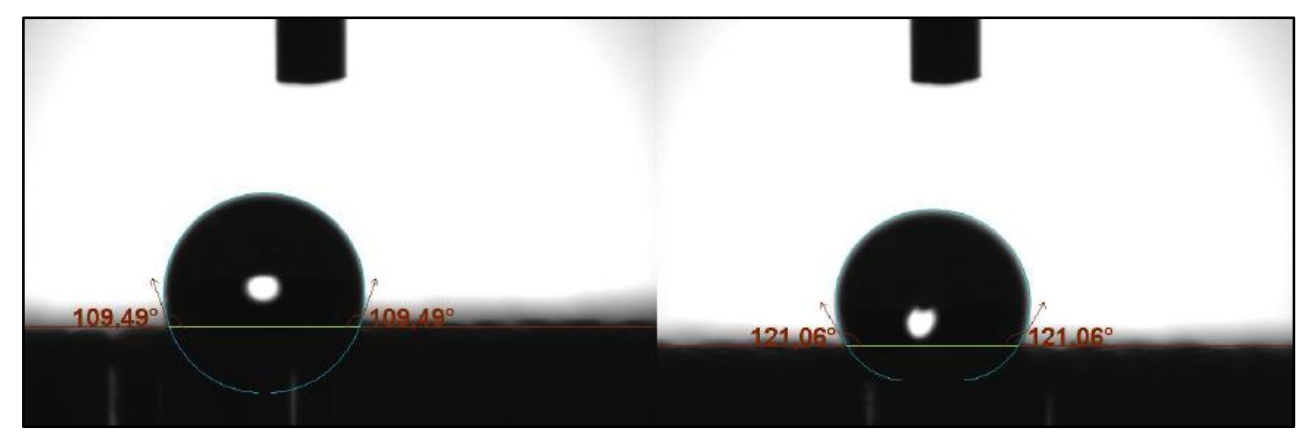

Figure 12: PM's contact angle measurement.

Then the static contact angle of PMR produced by replicating from a dental silicone material was approximately $100^{\circ}$ after 3 measurements (Figure 13).

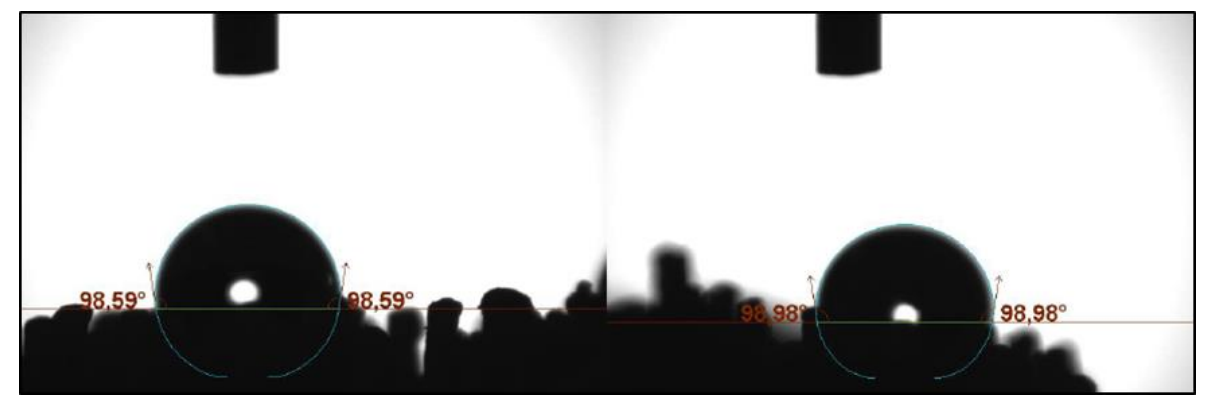

Figure 13: PMR's contact angle measurement.

The hydrophobic behavior of these microstructures showed also a sticky behaviour. Therefore, the water drop sticks to the microstructures and does not fall to the ground even when rotated $90^{\circ}$ or $180^{\circ}$ similar to that of the rose petal (Figure 14).

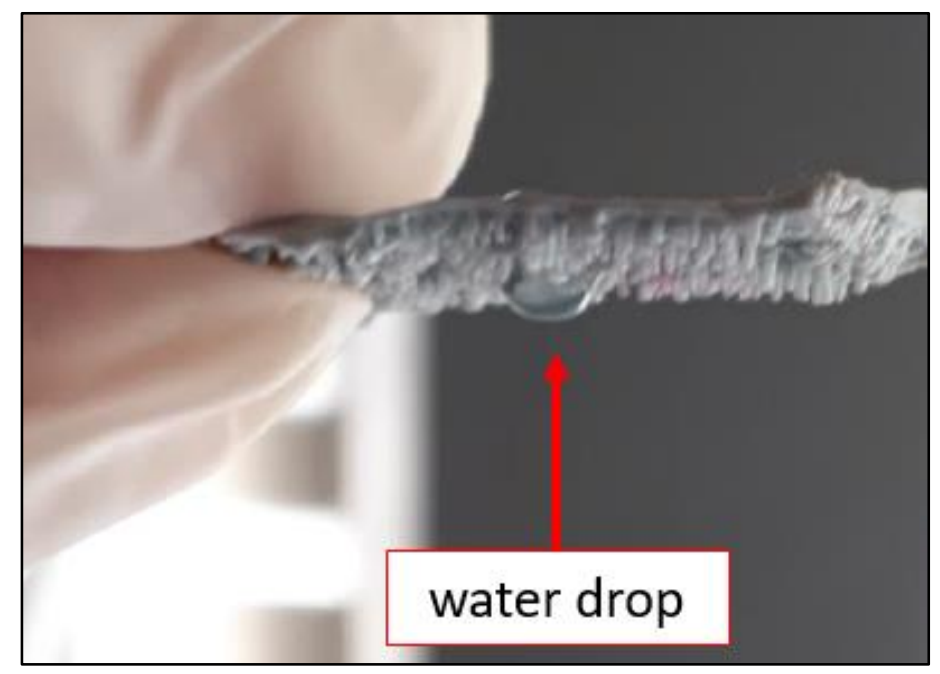

Figure 14: Water drop sticks to the surface when rotated $180^{\circ}$.

The surface of rose petals possesses hierarchical structures and exhibits superhydrobocity character. Moreover, a water drop placed on a red rose petal surface, stick with strong adhesion to the surface and the water drop does not roll-off even the surface is tilted $180^{\circ}$. The wettabillity behaviour of the rose petal was described as the "petal effect". Theoretically, 
superhydrophobicity is the result of the combination of micro- and nano-scale structures. Hence, we did not observe a superhydrophobic behaviour with these mushroom-shaped microstructures. It is also reported that rose petal effect can be achieved by introducing both nano- and microstructures [28]. In our case, we observed the rose petal effect on a singlescale microstructured surface.

On the other hand, the contact angle of the dental polymer structure replicated from the facial cleansing pad could not be measured because the water drop has spread among the conical needle-shaped pillar structures. This is an expected result because the pillars are in the millimeter scale and it cannot be expected to show superhydrophobic properties from pillars of this range.

In nature, the superhydrophobic property is possible by the hierarchical distribution of micro- and nano-structures. Therefore, only hydrophobic behaviour was observed with these conical shaped macro structures. The aim of producing such structıres was to increase the surface area when these pillars come in a contact with another surface.

\section{Conclusion}

In this study, pillars having different shapes and lengths were replicated successfully by soft molding from a dental polymer material. These replicated pillars which are from commercial products have high resolutions. The mushroom-tip pillars of the interlocking fastener were exhibited a rose-petal effect with only a single-scale structuring. Silicon facial cleansing pad is another replicated commercial product. The same bio-polymer material and method were used in the replicating process. In order to evaluate the effects of the length, orientation and shape of the replicated pillars on wettability a commercial product having macroscale conical shapes was also replicated with the same bio-polymer material. The replicated conical-shaped structures onto the bio-polymer material did not show any specific wettability property since its pillars are in the macroscale range. Since the pillars are also made of bio-materials, they are harmless to human health. These pillars having a petal effect can be further developed to potentially find applications for the transport of liquids, for improving light absorption and color saturation [29].

\section{References}

[1] Fu, H., Xiao, Q., Xing, J.D. (2008). A study on the crack control of a high-speed steel roll fabricated by a centrifugal casting technique. Materials Science and Engineering: A, 474(1-2): 82-87.

[2] Stefanescu, D. M., Davis, J. R., Destefani, J. D. (1988). Metals Handbook, Vol. 15 -Casting. ASM International, 1988, 937.

[3] Davis, J. R. (Ed.). (1996). ASM specialty handbook: cast irons. ASM international. p. 171.

[4] CES EduPack Software (2013), Granta Design Limited, Cambridge, UK.

[5] Wadsworth, J., Lesuer, D. R. (2000). Ancient and modern laminated composites-from the Great Pyramid of Gizeh to Y2K. Materials Characterization, 45(4-5):289-313. 
[6] Strnadel, B., Haušild, P. (2008). Statistical scatter in the fracture toughness and Charpy impact energy of pearlitic steel. Materials Science and Engineering: A, 486(1-2): 208-214.

[7] Zumelzu, E., Goyos, I., Cabezas, C., Opitz, O., Parada, A. (2002). Wear and corrosion behaviour of highchromium (14-30\% Cr) cast iron alloys. Journal of Materials Processing Technology, 128(1-3): 250-255.

[8] Singh, R. (2015). Applied welding engineering: processes, codes, and standards. Butterworth-Heinemann. p. 57-64.

[9] Marcuci, J. R. J., Souza, E. C. D., Camilo, C. C., Di Lorenzo, P. L., Rollo, J. M. D. A. (2014). Corrosion and microstructural characterization of martensitic stainless steels submitted to industrial thermal processes for use in surgical tools. Revista Brasileira de Engenharia Biomédica, 30(3): 257-264.

[10] Zumelzu, E., Goyos, I., Cabezas, C., Opitz, O., Parada, A. (2002). Wear and corrosion behaviour of highchromium (14-30\% Cr) cast iron alloys. Journal of Materials Processing Technology, 128(1-3): 250-255.

[11] Garrison Jr, W. M., Amuda, M. O. H. (2017). Stainless Steels: Martensitic. Reference Module in Materials Science and Materials Engineering

[12] Yousif I. F., Ataiwi A.H. (2018). Effects of heat treatment on erosion behavior and microstructure of high chromium white cast iron, Journal of Engineering and Applied Sciences, 13: 2376-2381

[13] Ataiwi, A. H. (2019). Study the Microstructure and Mechanical Properties of High Chromium White Cast Iron (HCWCI) under Different Martempering Quenching Mediums. Engineering and Technology Journal, 37(4part (A) Engineering), 112-119.

[14] Acton, Q. A. (2013). Iron Compounds-Advances in Research and Application: 2013 Edition: ScholarlyBrief. ScholarlyEditions. p. 197-199.

[15] Loto, R. T., Loto, C. A. (2017). Potentiodynamic polarization behavior and pitting corrosion analysis of 2101 duplex and 301 austenitic stainless steel in sulfuric acid concentrations. Journal of Failure Analysis and Prevention, 17(4): 672-679.

[16] El-Aziz, K. A., Zohdy, K., Saber, D., Sallam, H. E. M. (2015). Wear and corrosion behavior of high-Cr white cast iron alloys in different corrosive media. Journal of Bio-and Tribo-Corrosion, 1(4). 25.

[17] Jeong, H.E., Lee, J.K., Kim, H.N., Moon, S.H., Suh, K.Y. (2009). A Nontransferring Dry Adhesive with Hierarchical Polymer Nanohairs. Proceedings of the National Academy of Sciences, 106, 5639.

[18] Masuda, H., Satoh, M. (1996). Fabrication of Gold Nanodot Array Using Anodic Porous Alumina as an Evaporation Mask. Japanese Journal of Applied Physics, 35, Part 2.

[19] Thompson, G. (1997). Porous Anodic Alumina: Fabrication, Characterization and Applications. Thin Solid Films, 297, 192.

[20] Pashchanka, M., Schneider, J. J. (2011). Origin of Self-Organisation in Porous Anodic Alumina Films Derived from Analogy with Rayleigh-Bénard Convection Cells. Journal of Materials Chemistry, 21, 18761.

[21] Lee, W., Ji, R., Gösele, U., Nielsch, K. (2006). Fast Fabrication of Long-Range Ordered Porous Alumina Membranes by Hard Anodization. Nature Materials, 5: 741-747.

[22] Kustandi, T.S., Samper, V.D., Ng, W.S., Chong, A.S., Gao, H. (2007). Fabrication of a Gecko-Like Hierarchical Fibril Array Using a Bonded Porous Alumine Template. Journal of Micromechanics and Microengineering, 17, N75. 
[23] Ho, A.Y.Y., Yeo, L.P., Lam, Y.C., Rodríguez, I. (2011). Fabrication and Analysis of Gecko-Inspired Hierarchical Polymer Nanosetae. ACS Nano, 5, 1897.

[24] Lee, D.Y., Lee, D.H., Lee, S.G., Cho, K. (2012). Hierarchical Gecko-Inspired Nanohairs with a High Aspect Ratio Induced by Nanoyielding. Soft Matter, 8, 4905.

[25] Lee, H., Bhushan, B. (2012). Fabrication and Characterization of Hierarchical Nanostructured Smart Adhesion Surfaces. Journal of Colloid and Interface Science, 372, 231.

[26] Zhao,X.M., Xia, Y., Whitesides, G.M. (1997). Soft Litographic Methods for Nano-Fabrication, Journal of Materials Chemistry, 7(7): 1069-1074.

[27] Kroner E. K., (2011). Adhesion Measurements on Patterned Elastomeric Surfaces, der Universität des Saarlandes, 9-48.

[28] Feng, L., Zhang, Y., Xi, J., Zhu, Y., Wang, N., Xia, F., Jiang, L. (2008). Petal Effect: A Superhydrophobic State with High Adhesive Force. Langmuir 24(8): 4114-4119.

[29] Schulte, A.J. (2012). Light-trapping and superhydrophobic plant surfaces - optimized multifunctional biomimetic surfaces for solar cells, Ph.D. Thesis, Rheinische Friedrich Wilhelms Universität, Bonn, Germany. $113 \mathrm{p}$. 